Numer i cal anal ysi s of opt i cal feedback phenomenon and intensity noi se of fi bre-grat i ng semi conduct or I aser s

\begin{tabular}{|l|l|}
\hline 著者 & $\begin{array}{l}\text { Ahmed Noust af a, Nahnoud Saf wat W Z. , Yamada } \\
\text { M nor u }\end{array}$ \\
\hline $\begin{array}{l}\text { j our nal or } \\
\text { publ i cat i on t i t l e }\end{array}$ & $\begin{array}{l}\text { I nt er nat i onal J our nal of Numer i cal Nodel I i ng: } \\
\text { El ect r oni c Net wor ks, Devi ces and Fi el ds }\end{array}$ \\
\hline vol une & 20 \\
\hline number & 3 \\
\hline page r ange & $117-132$ \\
\hline year & $2007-05-01$ \\
\hline URL & ht t p: //hdl . handl e. net /2297/6596 \\
\hline
\end{tabular}




\title{
Numerical Analysis of Optical Feedback Phenomenon and Intensity Noise of Fiber-Grating Semiconductor Lasers
}

\author{
Moustafa Ahmed, `Safwat W. Z. Mahmoud \\ Department of Physics, Faculty of Science, Minia University, 61519 El-Minia, Egypt \\ Minoru Yamada \\ Division of Electrical Engineering and Computer Science, Graduate School on Natural Science \\ and Technology, Kanazawa University, Kakuma-machi, Kanazawa 920-1192, Japan.
}

\section{SUMMARY}

This paper demonstrates numerical analysis of the dynamics and intensity noise of fiber grating semiconductor lasers (FGSLs). The induced phenomenon of strong optical feedback (OFB) is analyzed. The simulations are based on an improved time-delay rate equations model of a single-mode laser that takes into account the multiple round trips of the lasing field in the fiber cavity. The analyses are performed in terms of the temporal trajectory of the laser intensity, bifurcation diagram and relative intensity noise (RIN). We explore influence of the fiber-cavity length on the dynamics and RIN. The results show that when the fiber-cavity is short, the regime of strong OFB is characterized by either continuous-wave $(\mathrm{CW})$ operation or periodic pulsation. The pulsation frequency is locked at the frequency separation of either the compound-cavity modes or the external fiber-cavity modes. The corresponding RIN level is close to or higher than the level of the solitary laser depending on pulse symmetry. When the fiber cavity is long, the laser exhibits unstable dynamics over wider range of OFB. Moreover, the strong-OFB pulsation becomes beating quasi-periodic at the relaxation oscillation frequency and the fiber-cavity mode-separation frequency.

KEY WORS: Chaos, fiber grating laser, intensity noise, optical feedback, pulsation, semiconductor laser.

- Corresponding Author:

Tel: 20-86-2339293, Fax: 20-86-2363011,Email: safwatwilliam@yahoo.com 


\section{INTRODUCTION}

Systems of FGSL's have been employed in several applications of fiber-optic communications. As illustrated in the scheme of Fig. 1, a FGSL consists of a semiconductor laser with anti-reflection coating (ARC) on the front facet and highreflection coating (HRC) on the back facet. The laser is coupled with an external fiber cavity formed between the front facet and a fiber grating (FG). When subjected to intensity modulation, the FGSL, due to its structure, helps to lock the lasing mode stimulating the laser to operate in the so called "active mode-locking" [1,2]. These activemode locked lasers can be used as sources of short optical pulses with large locking range at a fixed control wavelength for use in high-speed optical communications [3]. Another typical application of FGSLs is the use as pumping sources in optical fiber amplifiers that are employed to compensate weakness of the transmitted optical signal along optical fibers in communication systems [4]. In such applications, the FGSL structure helps to fix the pumping wavelength at the FG peaked wavelength and to increase the pumping power. Due to the very low reflection coefficient $\left(\sim 10^{-4}\right)$ of the AR-coated facet, the laser operates with an external fiber cavity of strong OFB at the lasing wavelength [5,6]. Recent investigations of the operation characteristics of these lasers have indicated that the device may operate in $\mathrm{CW}$ or pulsation (periodic or quasi-periodic) even when injected with dc current, depending on the lasing conditions and the system configuration [7,8]. A vital issue of FGSLs is the intensity noise on the emitted signal which measures performance of the application systems [4]. An important design parameter of the FGSL is the length of the external fiber cavity, which should be optimized to emit laser radiation with minimum intensity noise and stable spectrum. Most previous studies of lasers under OFB relied on the Lang-Kobayashi model, which treats the OFB as a perturbation of the electric field due to a round trip in the external cavity [9]. Although this model is suitable to weak and 
rather moderate OFB, appropriate modifications to adopt it for cases of strong OFB have received little attention [7,10-12].

In [7] an improved model of semiconductor lasers working under OFB has been proposed basing on a time-delay rate equation model of both the intensity and phase of the electric field and the number of injected electrons. A typical feature of that model is the ability of analyzing laser dynamics under an arbitrary OFB strength ranging from weak of very strong OFB [7,13]. The model has been successfully applied to investigate the dynamic characteristics and RIN of semiconductor lasers coupled with a long fiber cavity $[7,8,14]$. It was shown that the laser works under stable $\mathrm{CW}$ operation with suppressed relative intensity noise (RIN) in the weak OFB regime. When the OFB strength increases, the laser suddenly enters a region of chaos characterized by enhanced levels of RIN. This chaotic dynamic characterizes the regimes of intermediate and rather strong OFB [7,8,14]. Further increase of the OFB strength was found to bring the laser into a regime of frequency locking with the locked-frequency being determined by the fiber-cavity length [7]. Influence of the length of the external cavity on the FGSL dynamics, however, has not been considered.

In this paper, we simulate the operation characteristics, dynamics and spectral profiles of RIN of 980nm-InGaAs FGSL. We are aimed at exploring the OFB parameters that correspond to stable operation and low RIN; Namely, the relative reflectivity of the FG compared with the reflectivity of the ARC-front facet, and the length of the fiber cavity. We consider both cases that the fiber cavity length corresponds to an external-cavity mode-frequency separation higher and lower than the relaxation oscillation frequency. The analyses are made in terms of the temporal trajectory of the photon number, the corresponding bifurcation diagram and the spectrum of the RIN. The results show that the laser operation is almost periodic with frequency locking at the compound-cavity mode-separation frequency or the external-cavity mode-separation frequency under strong OFB depending on the length of the fiber cavity. When the fiber cavity is short, the 
operation is almost stable characterized by either pulsation or continuous wave, and the route-to-chaos is almost period doubling. The regime of long-fiber cavity is characterized by high levels of low-frequency RIN and a quasi-periodic route-to-chaos. Lengthening the fiber cavity results in further promotion of the low-frequency components of RIN.

The paper is structured as follows. The simulation model of laser dynamics and RIN is given in the next section. Section 3 presents the procedures of applying the proposed model in computer simulation. In section 4 , we demonstrate variation of the power emitted from the FG with OFG strength, and the simulation results of laser dynamics and RIN under different lengths of the fiber cavity. The conclusions are given in section 5 .

\section{COMPUTER SIMULATION MODEL}

The proposed model of analysis of OFB of a FGSL is schematically illustrated in Fig. 2. The light emitted from the laser front facet at a time $t$ travels multiple round-trips in the fiber cavity. This cavity is characterized by the reflectivities $R_{f}$ and $R_{G}$ of the ARC-facet and the FG. During each round trip, the time delayed laser light reflected by the FG injects into the laser cavity through the front facet. The time duration of each round-trip is $\tau=2 n_{F} L_{F} / c$, with $L_{F}$ and $n_{F}$ as the length and refractive index of the fiber cavity, and $c$ as the speed of light in vacuum. Therefore at a time $t$ at the front facet, the photon number $S(t)$ inside the cavity is influenced by the time-delayed photon numbers $S(t-m \tau)$, where $m=1,2,3, .$. is a roundtrip index. Influence of the phenomenon of OFB on laser dynamics is described by the following time-delay rate equations of the photon number $S(t)$, optical phase $\theta(t)$ and injected electron number $N(t)[7,13]$ :

$$
\frac{d S}{d t}=\left(A-B S-G_{t h 0}+\frac{c}{n_{D} L_{D}} \ln |U|\right) S+\frac{a \xi}{V} N+F_{S}(t),
$$




$$
\begin{aligned}
& \frac{d \theta}{d t}=\frac{1}{2}\left[\frac{\alpha a \xi}{V}(N-\bar{N})-\frac{c}{n_{D} L_{D}} \varphi\right]+F_{\theta}(t), \\
& \frac{d N}{d t}=-A S-\frac{N}{\tau_{S}}+\frac{I}{e}+F_{N}(t),
\end{aligned}
$$

where $A$ and $B$ are linear and nonlinear coefficients of optical gain $G_{D}$, respectively. They are practically given as linear functions of the electron number $N$ [15],

$$
\begin{aligned}
& A=\frac{a \xi}{V}\left(N-N_{g}\right), \\
& B=B_{c}\left(N-N_{S}\right) .
\end{aligned}
$$

The term $a \xi N / V$ in Eq. (1) indicates the rate of inclusion of the spontaneous emission into the lasing mode. The parameters in the above equations are defined as follows.

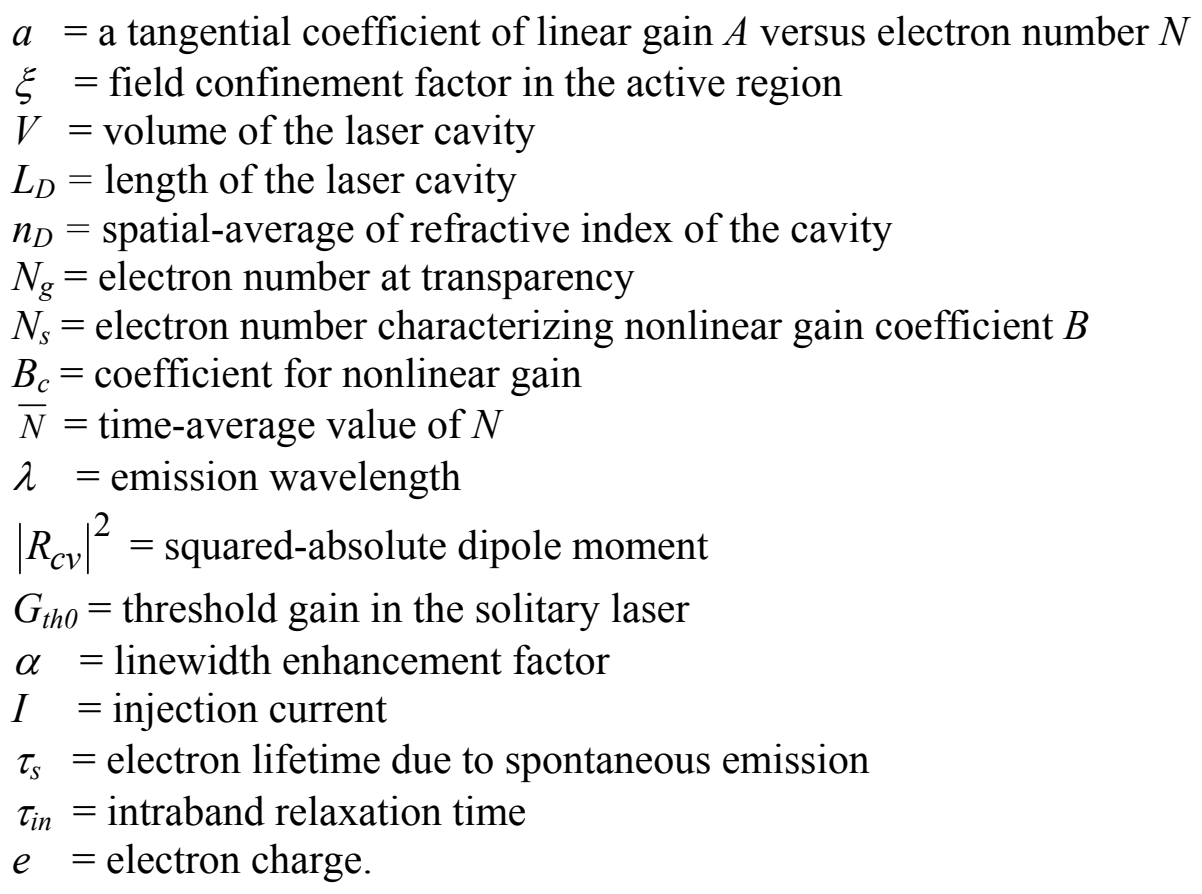

The OFB is counted in terms of the function $U$ and its argument $\varphi$, which are defined in terms of the round-trip time $\tau$ as [13]: 


$$
\begin{aligned}
U & =1-\sum_{m=1}\left(K_{e x}\right)^{\mathrm{m}}\left(\frac{R_{f}}{1-R_{f}}\right)^{m-1} \exp \{-j m \psi\} \frac{S(t-m \tau)}{S(t)} \\
& =|U| \exp \{-j \varphi\},
\end{aligned}
$$

where $\psi$ is a phase term combining the phase changes due to reflection by the FG, $\phi_{G}$, and front facet, $\phi_{f}$, and due to a round-trip in the fiber cavity, $\omega \tau$ with being the circular frequency of the emission;

$$
\psi=\phi_{G}+\phi_{f}+\omega \tau
$$

The strength of OFB is measured by the coefficient $K_{e x}$, which is determined by the ratio of FG reflectivity $R_{G}$ to the front-facet reflectivity $R_{f}$ as,

$$
K_{e x}=\left(1-R_{f}\right) \sqrt{\eta R_{G} / R_{f}}
$$

where $\eta$ is the coupling ratio of the externally reflected light into the laser cavity. The argument $\varphi$ of the complex feedback function $U$ is obviously given by:

$$
\varphi=-\tan ^{-1}\{\operatorname{Im}[U] / \operatorname{Re}[U]\}+\ell \pi
$$

where $\ell$ is an integer. Determining the value of $\varphi$ in the two-dimensional space $(\operatorname{Re}[U]-\operatorname{Im}[U])$ depends on both the signs and magnitudes of $\operatorname{Re}[U]$ and $\operatorname{Im}[U]$.

The terms $F_{S}(t), F_{\theta}(t)$ and $F_{N}(t)$ are Langevin noise sources of a Gaussian type with zero means, and satisfy the following cross-correlations $[16,17]$ :

$$
\left\langle F_{a}(t) F_{b}\left(t^{\prime}\right)\right\rangle=V_{a b} \delta\left(t-t^{\prime}\right)
$$

where $a$ and $b$ stand for either $S, N$ or $\theta$. These random terms are added to the rate equations to account for the quantum noises associated with inclusion of the spontaneous emission and recombination processes of charge carriers into the lasing process. The correlation 
variances $V_{a b}$ are determined from the steady-state solutions $\bar{S}$ and $\bar{N}$ of Eqs. (1)-(3).

The noise content of the photon number fluctuations is determined in terms of the RIN.

The RIN is calculated from the fluctuations $\delta S(t)=S(t)-\bar{S}$ of $S(t)$ via the following equation [16]:

$$
R I N=\frac{1}{\bar{S}^{2}}\left\{\frac{1}{T}\left|\int_{0}^{T} \delta S(\tau) e^{-j \Omega \tau} d \tau\right|^{2}\right\}
$$

where $\Omega$ is the Fourier frequency and $\bar{S}$ is the time-average value of $S(t)$.

In applications of the FGSL as a pumping source of an optical fiber amplifier, it is important to examine influence of the external OFB on the power emitted from the fiber grating $P_{G}$. This power is calculated based on a traveling wave model of the lasing field in the laser and external cavities as [7],

$$
\begin{aligned}
& P_{G}(t)=\frac{h c^{2}}{2 n_{D} \lambda L_{D}} S(t) \\
& X \frac{\frac{1}{2} \ln \left(\frac{1}{R_{f} R_{b}}\right)+\ln \left(\frac{1}{|U|}\right)}{1+\left[\frac{1}{\sqrt{R_{b}}}-\sqrt{R_{b}}\right] \sqrt{R_{f}}|U|-|U|^{2} R_{f}}\left(1-R_{f}\left(\frac{K_{e x}}{1-R_{f}}\right)^{2}\right)\left(1-R_{f}\right)|X|^{2}
\end{aligned}
$$

where $X$ is another OFB function describing transmission of the lasing field in the external fiber cavity, and is given by.

$$
X=\sum_{m=0}\left(K_{e x}\right)^{\mathrm{m}}\left(\frac{R_{f}}{1-R_{f}}\right)^{m} \exp \{-j m \psi\} \frac{S(t-m \tau)}{S(t)} .
$$




\section{PROCEDURES OF NUMERICAL CALCULATIONS}

In the present calculations, we employ parameters of a $980 \mathrm{~nm}$ InGaAs fiber grating laser shown in Fig. 1. Typical values of such parameters are listed in Table I. At a give injection current level $I$, rate equations (1)-(3) are solved numerically by means of the fourth-order Runge-Kutta method. The time step of integration is set as short as $\Delta t=5 \mathrm{ps}$ to guarantee fine resolution of the OFB-induced dynamics. This small time step $\Delta t$ corresponds to a cut-off frequency much higher than the external-cavity mode-separation frequency $f_{\text {ex }}=1 / \tau$. Three roundtrips, $m=1,2,3$, are counted in the calculations. Counting more roundtrips was found not to affect the simulated laser dynamics. The integration is first solved without OFB (the case of the solitary laser) from time $t=0$ until the first round trip time $\tau$. The calculated values of $S(t=0 \rightarrow \tau)$ and $\theta(t=0 \rightarrow \tau)$ are then stored for use as time delayed values $S(t-\tau)$ and $\theta(t-\tau)$ for integration of the rate equations over the period $t=\tau \rightarrow 2 \tau$ including OFB terms. Then the calculated values $S(t-\tau), S(t-2 \tau), \theta(t-\tau)$ and $\theta(t-2 \tau)$ are used as time delayed values for integration over the period $t=2 \tau \rightarrow 3 \tau$. This process continues for the third round trip. The integration is then proceeded over a long period of time $T=3 \sim 4 \mu$ s during which the operation state is stabilized [8] by considering all terms of $S(t-m \tau)$ and $\theta(t-m \tau)$ as time delayed values. The combined phase $\psi$. is arbitrary and is set to zero in the present calculations. The RIN is then computed directly from the obtained values of $S(t)$ by employing the fast Fourier transform (FFT) to integrate the discrete versions of Eq. (11) as:

$$
R I N \equiv \frac{1}{\bar{S}^{2}} \frac{\Delta t^{2}}{T}\left|F F T\left[\delta S\left(t_{i}\right)\right]\right|^{2},
$$

In order to perform the above calculations, discrete generations of the noise sources $F_{S}\left(t_{i}\right), F_{S}\left(t_{i}\right)$ and $F_{\theta}\left(t_{i}\right)$ at each instant $t_{i}$ are necessary. These processes require enough care in order to satisfy the mutual cross-correlations among the noise source at each time $t_{i}$. These cross-correlations are manifests of the cross-correlation between $S$ and $N$ during the 
lasing action. In this paper, we follow the self-consistent technique proposed by Ahmed et al. $[16,17]$ to adopt such generation processes. The obtained forms of the noise sources are:

$$
\begin{aligned}
& F_{S}\left(t_{i}\right)=\sqrt{\frac{V_{S S}\left(t_{i}\right)}{\Delta t}} g_{S}, \\
& F_{\theta}\left(t_{i}\right)=\frac{1}{S\left(t_{i}-1\right)+1} \sqrt{\frac{V_{S S}\left(t_{i}\right)}{\Delta t}} g_{\theta}, \\
& F_{N}\left(t_{i}\right)=\sqrt{\frac{V_{N N}\left(t_{i}\right)+2 k_{S}\left(t_{i}\right) V_{N S}\left(t_{i}\right)}{\Delta t}} g_{N}-k_{S}\left(t_{i}\right)\left\{F_{S}\left(t_{i}\right)+2\left[S\left(t_{i-1}\right)+1\right] F_{\theta}\left(t_{i}\right)\right\},
\end{aligned}
$$

with

$$
\begin{aligned}
V_{S S}\left(t_{i}\right) & =2 \frac{a \xi}{V}\left[S\left(t_{i-1}\right)+1\right] N\left(t_{i-1}\right), \\
V_{N N}\left(t_{i}\right) & =2\left[\frac{1}{\tau_{S}}+\frac{a \xi}{V} S\left(t_{i-1}\right)\right] N\left(t_{i-1}\right), \\
V_{N S}\left(t_{i}\right) & =-\frac{a \xi}{V} N\left(t_{i-1}\right)\left[S\left(t_{i-1}\right)+1\right]+N_{g} S\left(t_{i-1}\right), \\
k_{S}\left(t_{i}\right) & =-\frac{V_{N S}\left(t_{i}\right)}{V_{S S}\left(t_{i}\right)} .
\end{aligned}
$$

In the above equations, $g_{S}, g_{\theta}$, and $g_{N}$ are three independent Gaussian random numbers with means of zero and variances of unity. They are obtained at each integration step by applying the Box-Mueller approximation [18] to a set of three uniformly distributed independent random numbers generated by the computer. 


\section{SIMULATION RESULTS AND DISCUSSION}

\section{1. $C W$-based study of influence of OFB parameters on power emitted from $F G$}

We elucidate dependency of the power $P_{G}$ emitted from the FG on OFB parameters; namely, OFB strength $K_{e x}$ and fiber cavity length $L_{F}$, by assuming CW operation of the laser. The CW operation (steady state solutions of rate equations (1)-(3)) is obtained on the condition that the photon number $S(t)$ inside the laser cavity and time-delayed number $S(t-m \tau)$ are the same,

$$
S(t-m \tau)=S(t)
$$

By setting the combined phase as $\psi=\omega \tau$, i.e., phase change of the lasing field due to a round-trip in the fiber cavity, the summations of $U$ and $X$ in Eqs. (6) and (13) are collected as,

$$
\begin{gathered}
U_{c}=\frac{1-R_{f}-K_{e x} \exp \{j \omega \tau\}}{1-R_{f}-R_{f} K_{e x} \exp \{j \omega \tau\}} \\
X_{c}=\frac{1-R_{f}}{1-R_{f}-R_{f} K_{e x} \exp \{j \omega \tau\}}
\end{gathered}
$$

The threshold gain under OFB then becomes:

$$
G_{t h c}=G_{t h 0}-\frac{c}{n_{D} L_{D}} \ln \left|U_{c}\right|
$$

which determines the injected electron number $N_{t h c}$ and injection current $I_{t h c}$ at threshold as:

$$
\begin{gathered}
N_{t h c}=N_{g}+\frac{V}{a \xi} G_{t h c} \\
I_{t h c}=\frac{e N_{t h c}}{\tau_{s}}
\end{gathered}
$$

At an injection current above threshold, $I>I_{\text {thc }}$, the photon number in the laser output is well 
approximated as

$$
S_{c}=\frac{I-I_{t h c}}{e G_{t h c}}
$$

The CW emitted power $P_{G c}$ is then calculated via Eq. (12) using $S_{c}, U_{c}$ and $X_{c}$. Figs. 3(a) and (b) plot variations of $P_{G c}$ with the OFB strength $K_{e x}$ and fiber cavity length $L_{F}$, respectively, when $I=5 I_{t h 0}$ with $I_{t h 0}$ as the threshold current of the solitary laser. Fig. 3(a) corresponds to fiber cavity lengths of $L_{F}=5,20$ and $50 \mathrm{~cm}$. The figure shows that for $L_{F}=5$ and $50 \mathrm{~cm}$, the increase of OFB strength is associated with slow increase of $P_{G c}$ in the weak OFB regime followed by a fast increase in the strong regime up to the vicinity of $K_{e x} \approx 7$. On the other hand, when $L_{F}=20 \mathrm{~cm}, P_{G c}$ decreases with the increase of $K_{e x}$ up to $K_{e x}=0.95$ at which $P_{G c}$ changes to increase up to the vicinity of $K_{e x} \approx 7$. At this particular OFB strength, the term $R_{f}\left(K_{e x} /\left(1-R_{f}\right)\right)^{2}$ in Eq. $(12)$ becomes unity and $P_{G c}$ becomes zero. In the vicinity of the critical value of $K_{e x}$, the threshold gain $G_{t h c}$ approaches zero. This critical value of $K_{e x}$ shifts toward weaker OFB with the increase of $R_{f}$. Fig. 3(a) shows also that further increase of $K_{e x}$ results in sharp increase of the power $P_{G c}$ over a narrow range followed by a rapid decrease. On conclusion, the strong OFB regime corresponds to effective increase of $P_{G c}$ with the variation of OFB strength. Fig. 3(b) illustrates the influence of fiber cavity length $L_{F}$ on $P_{G c}$ at particular OFB strengths of $K_{e x}=0.2$ and 2 . The figure plots the variation of $P_{G c}$ with $L_{F}$ over an optical length $n_{F} L_{F}$ equal to five orders of the emission wavelength. The figure shows the property of periodic variation of $P_{G c}$ with variation of $L_{F}$ as a manifest of the standing-wave pattern formed in the fiber cavity; $P_{G c}$ is maximum when $n_{F} L_{F}$.is an odd number of $\lambda / 4$ and minimum when $n_{F} L_{F}$. is an even number of $\lambda / 4$. The amplitude of power oscillation is higher for $K_{e x}=2$ than for $K_{e x}=0.2$ as inferred from Fig. 3(a). 


\subsection{Short fiber-cavity: time variation of laser intensity and frequency spectra of RIN}

Fig. 4 plots the simulation results of the photon number $S(t)$ and RIN characterizing possible laser operations under OFB in the limit of short fiber cavity, $L_{F}=1 \mathrm{~cm}$. The injection current $I$ is set well above the threshold, $I=5 I_{t h 0}$. This case of short fiber cavity corresponds to the case that the external cavity mode frequency separation $f_{e x}=c / 2 n_{F} L_{F}=10 \mathrm{GHz}$ is higher than the frequency of the relaxation oscillation, $f_{r} \approx 4.1 \mathrm{GHz}$. Figs. 4 (a)-(e) plot the temporal trajectories of $S(t)$, while Figs. 3(f)-(j) plot the corresponding Fourier-frequency spectra of RIN. The RIN spectrum of the solitary laser is also plotted in Figs. 4(f)-(j) with dashed lines for comparison. Figs. 4(a) and (f) correspond to the CW operation characterizing the weak regime of OFB, $K_{e x}=0.03$. Fig. $4(\mathrm{a})$ shows that the emitted photon number $S(t)$ fluctuates around its average value $\bar{S}$. The amplitude of the fluctuations is comparable to that of the solitary laser. Fig. 4(f) shows that the RIN spectrum is almost coincident with that of the solitary laser exhibiting the pronounced peak at $f_{r}$. This means that the OFB is not strong enough to change the relaxation oscillation of the laser.

Figs. 4(b) and (g) correspond also to the weak OFB regime but with stronger OFB, $K_{e x}=0.07$. The figures correspond to pulsing operation; Fig. 4(b) shows regular pulsation with duration of $1 / f_{r}$. These pulses are generated because the OFB becomes strong enough to reduce the damping rate of the laser and undamp the relaxation oscillation. The RIN spectrum shown in Fig. 4(g) exhibits very high and sharp peaks around $f_{r}$ and its higher harmonics. Compared with the RIN spectrum of the solitary laser, the low frequency (LF) part of the RIN spectrum is more flat (white noise) and higher (more than two orders of magnitude). This increase of RIN is because the laser pulses have sharp peaks but are broaden at the tails.

Figs. 4(c) and (h) plot the most noisy operation suffered by the laser in the intermediate range of OFB, $K_{e x}=0.21$. This operation state is known as chaos and is associated with 
extreme broadening of the laser spectral line [13,19]. Fig. 4(c) indicates irregular time variation of $S(t)$ with the time variation. Fig. 4(h) shows that the entire RIN spectrum is about four-orders of magnitude higher than that of the solitary laser, which is a typical feature of chaotic operation $[12,13,20,21]$. The high-frequency peaks of the RIN spectrum are well suppressed compared with those of the pulsing operation.

Figs. 4(d) and (i) characterize another type of regular pulsation induced in the regime of strong OFB, $K_{e x}=2.57$. Compared with the weak OFB-induced pulsation in Fig. 4(d), the pulsation becomes faster. The pulse duration is nearly $1 / f_{\mathrm{c}}$ with $f_{c}=c /\left(2 n_{D} L_{D}+2 n_{F} L_{F}\right)$ as the frequency separation of the compound cavity modes (combining both the laser and external cavities). This result is confirmed by the frequency spectrum of RIN of Fig. 4(i) which exhibits very sharp and high two peaks around $f_{c}$ and its second-order harmonic. This effect indicates that the laser follows the resonance condition of the compound cavity rather than the laser cavity itself. The LF-part of the RIN spectrum is nearly two orders of magnitude higher than that of the solitary laser; it is lower than the level characterizing the weak OFB-induced pulsation shown in Fig. 4(g).

Figs. 4(e) and (j) characterize the CW operation exhibited in the regime of strong OFB, $K_{e x}=4.0$. Fig. $4(\mathrm{e})$ shows that the amplitude of fluctuations of $S(t)$ is comparable to that of the solitary laser. Fig. 4(i) shows that the LF-part of the RIN spectrum is almost coincident with that of the solitary laser. However, the high-frequency regime of the RIN spectrum is characterized by a weak peak around $f_{e x}$ not around $f_{r}$ that distinguishes the $\mathrm{CW}$ operation induced under weak OFB.

\subsection{Short Fiber-Cavity: Bifurcation diagram and low-frequency RIN}

In this subsection we numerically classify the influence of the OFB on the laser dynamics in terms of the bifurcation diagram. The bifurcation diagram is a convenient 
method to analyze the laser dynamics and explore the OFB range corresponding to possible states of laser operation [20]. The diagram is calculated numerically at a constant current of $5 I_{\text {tho }}$, and is constructed by picking up the peaks $S_{\text {peak }}(t)$ of the time-varying photon number $S(t)$ normalized by the time-average value $\bar{S}$ at each OFB strength $K_{e x}$. It has been common to drop the Langevin noise sources in such calculations. However it has been shown that the intrinsic fluctuations of $S, \theta$, and $N$ represented by the instantaneous generations of these noise sources may dramatically change the state of laser output [8]. In the present calculations, we include such random generations seeking more general analysis. The simulated bifurcation diagram is shown in Fig. 5(a). The figure shows that the laser still operates stably in CW under small values of $K_{e x}$. These CW dynamics are plotted in the figures as points with small values of $S_{\text {peak }}$ that represent small fluctuations of $S(t)$ around $\bar{S}$. At a certain value of $K_{e x}=0.043$, which represents a Hopf-bifurcation point, the laser output becomes periodic as the relaxation oscillations become undampled. The result is then plotted as a single point in the bifurcation diagram (or several closed points that represent fluctuations of $S_{\text {peak }}(t)$ as manifest of the Langevin noise sources). As discussed above, this pulsation is characterized with the relaxation oscillation frequency $f_{r}$. At another critical OFB strength $K_{e x} \approx 0.078$, the point last point of periodic pulsation bifurcates into two points indicating that the laser emits pulses with period doubling. The pulsation in the period doubling region $\left(K_{e x}=0.078 \sim 0.088\right)$ is characterized with the sub-harmonic frequency $f_{r} / 2$. With further increase of $K_{e x}$, bifurcation into multiple paths occurs enhancing dynamic irregularities in the laser output and bring the laser into chaotic dynamics. This description is known as period-doubling route-to-chaos and characterizes the case of short-external cavity case [21]. The chaos region ends when $K_{e x} \approx 0.22$ at which the laser changes suddenly to $\mathrm{CW}$ operation. By further increase of the OFB strength, the laser enters a region of frequency locking at which the laser operates with regular pulsation as characterized in Figs. 
4(d) and (i). The most far points of this region (with strongest OFB) are characterized with $f_{e x}$ as a locking frequency.

In Fig. 5(b), we plot the corresponding variation of the LF-RIN averaged over the Fourier-frequencies $f<100 \mathrm{MHz}$. The figure shows that the regions of $\mathrm{CW}$ operation are characterized with lowest LF-RIN levels $(-175 \mathrm{~dB} / \mathrm{GHz})$, while the region of chaotic operation $\left(K_{e x}=0.11 \sim 0.22\right)$ is described by the most enhanced LF-RIN levels $(-155 \sim-135 \mathrm{~dB} / \mathrm{GHz})$. The regions of periodic pulsation in either weak or strong OFB limit are characterized in general by high LF-RIN levels in the range $(-165 \sim-155 \mathrm{~dB} / \mathrm{GHz})$. However, at some OFB strengths the LF-RIN levels approach the level of the CW operation. In such cases, the produced pulses are symmetric with respect to the mean photon number.

\subsection{Influence of Fiber-Cavity Length on Laser dynamics}

We also use the bifurcation diagram to study influence of the length of the fiber cavity $L_{F}$ on the laser dynamics at arbitrary strength of the OFB. The simulated bifurcation diagrams corresponding to different cavity lengths of $L_{F}=10,20$ and $80 \mathrm{~cm}$ are shown in Fig. 7(a)-(c), respectively. When the fiber length $L_{F}$ is $10 \mathrm{~cm}$, Fig. 6(a) shows that the period doubling disappears and the route-to-chaos becomes quasi-periodic because $f_{e x}$ becomes $1 \mathrm{GHz}$ which is lower than $f_{r} \approx 4.1 \mathrm{GHz}$ [21]. In this case the range of OFB strength, $K_{e x} \approx 0.043 \sim 0.056$, over which the laser emits regular pulsation on the route-to-chaos becomes narrow compared with Fig. 5(a) of $L_{F}=1 \mathrm{~cm}$. In Fig. 6(b) of $L_{F}=20 \mathrm{~cm}$, the route-to-chaos can still be seen and the range of $K_{e x}$ corresponding to regular pulsation becomes much narrower. When $L_{F}$ becomes $80 \mathrm{~cm}$ Fig. 6(c) shows that the quasi-route-to-chaos hardly appears and the chaos decade becomes wider than the cases of shorter fiber cavities; it extends over $K_{e x} \approx 0.05 \sim 0.37$. Following this chaos decade, another chaotic operation is seen around $K_{e x}=0.52$. Therefore, the longer the fiber cavity is, the 
wider is the range of OFB over which the RIN is enhanced. Another interesting feature associated with increasing $L_{F}$ is that the region of frequency locking characterizing the strong-OFB regime of $L_{F}=1 \mathrm{~cm}$ is no longer periodic with the increase of $L_{F}$. Instead, the laser follows a route to quasi-periodicity with beating pulsation. This is seen as three points of $S_{\text {peak }(t)}$ in the bifurcation diagram of Fig. $6(\mathrm{c})$ of $L_{F}=100 \mathrm{~cm}$, and two points in the diagrams Fig. 6(a) and (b) of shorter cavities $L_{F}=10$ and $20 \mathrm{~cm}$, respectively.

In Fig. 7 we characterize the temporal trajectory of $S(t)$ and the RIN spectrum of this route-to-periodicity induced under strong OFB when $L_{e x}=20 \mathrm{~cm}$. Figs. 7(a) and (b) plot the temporal trajectories of $S(t)$, while Figs. 7(c) and (d) plot the corresponding RIN spectra. Fig. 7(a) and (c) show that when $K_{e x}=3$, the laser operates in regular pulsation at $f_{r}$ in similar fashion to the route-to the weak-OFB chaos decade. The RIN spectrum shown in Fig. 7(c) exhibits very high and sharp peaks around $f_{r}$ and its second harmonic. The LF-part of RIN is little higher than that of the regular pulsations induced under both weak and strong OFB of Figs. 4(g) and (i), respectively. Fig. 7(b) shows that when $K_{e x}$ increases to 3.1, the laser dynamics becomes quasi-periodic with two peaks characterizing beating pulsation. The peaks of equal and higher peaks have fine time duration of $1 / f_{r}=0.25 \mathrm{~ns}$, while the lower peaks have wider duration of $1 / f_{e x}=2 \mathrm{~ns}$. This behavior is confirmed in the RIN spectrum of Fig. 7(d), which shows sharp peaks at $f_{e x}=500 \mathrm{MHz}$ and its higher harmonics as well as a broad peak around the relaxation frequency $f_{r} \approx 4.1 \mathrm{GHz}$. Fig. $7(\mathrm{~d})$ shows also that the LF-RIN becomes lower than the case of $K_{e x}=3$, but is still higher than that of the solitary laser. 


\section{CONCLUSIONS}

We numerically characterized the dynamics and RIN of FGSLs. Influences of OFB and fiber-cavity length are demonstrated basing on a model of time-delay rate equations. The analyses are achieved by means of the bifurcation diagram including the Langevin noise sources. Based on the simulated results, we can trace the following conclusions.

(1) Regardless of the fiber-cavity length, the laser operates in CW or pulsation under weak OFB and chaos under intermediate OFB. The route-to-chaos is period doubling for short fiber cavities and quasi-periodic for long cavities.

(2) Under chaotic dynamics, the low-frequency RIN level is almost four orders of magnitude higher than that of the solitary laser

(3) When the fiber-cavity is short, the regime of strong OFB is characterized by either $\mathrm{CW}$ operation or regular pulsation with the pulsation frequency locked at the frequency separation of the modes of either the compound or fiber cavity.

(4) When the fiber-cavity is long, the pulsation becomes quasi-periodic of a beating type characterized by RIN levels two-orders of magnitude higher than that of the solitary laser.

(5) The RIN level of the laser under pulsing dynamics is close to or higher than that of the solitary laser depending on symmetry of the pulses. 


\section{REFERENCES}

1. Pittoni F, Gioannini M, Montrosset I. Time-domain analysis of fiber grating semiconductor laser operation in active mode-locked regime. IEEE Journal of Selected Topics on Quantum Electronics 2001; 7(2): 280-286.

2. Lowery AJ, Marshall IW. Numerical simulation of $1.5 \mu \mathrm{m}$ actively mode-locked semiconductor lasers including dispersive elements and chirp. IEEE Journal of Quantum Electronics 1991; 27(8): 1981-1989.

3. Paoletti R, Bertone D, Fang R, Magnetti G, Meliga M, Meneghini G, Morello G, Rossi G, Tallone L, Scofet M. $1.55 \mu \mathrm{m}$ optical short pulse generation at $10 \mathrm{GHz}$ repetition rate using a mode-locked hybrid distributed Bragg reflector (ML-HDBR) lasers. IEEE Photonics Technology Letters 2000; 12(3): 245-247.

4. Agrawal GP. Optical Fiber Communication Systems, Chapter ㅁ. Van Nostrand Reinhold: New York, 2003.

5. Mokai T, Otsuka K. New route to optical chaos: Successive-SubharmonicOscillation Cascade in a semiconductor laser coupled to an external cavity. Physical Review 1985; 55(17-21): 1711-1714.

6. Hohl A, Gavrielides A. Bifurcation cascade in a semiconductor laser subject to optical feedback. Physical Review Letters 1999; 82(6): 1148-1151.

7. Abdulrhmann S, Ahmed M, Okamoto T, Ishimori W, Yamada M. An improved analysis of semiconductor laser dynamics under strong optical feedback. IEEE Journal of Selected Topics on Quantum Electronics 2003; 9(5): 1265-1274.

8. Abdulrhmann S, Ahmed M, Yamada M. New model of analysis of semiconductor laser dynamics under strong optical feedback in fiber communication systems. SPIE 2003; 4986: 490-501.

9. Lang R, Kobayashi K. External optical feedback effects on semiconductor injection laser properties. IEEE Journal of Quantum Electronics 1980; QE-16(3): 347-355.

10. Rong-Qing $\mathrm{H}$, Shang-Ping T. Improved rate equations for external cavity semiconductor lasers. IEEE Journal of Quantum Electronics 1989; 25(6): 15801584.

11. Langley LN, Shore KA, Mork J. Dynamical and noise properties of laser diodes subject to strong optical feedback. Optics Letters 1994; 19: 2137-2139.

12. Kallimani KI, O’Mahony MJ. Relative intensity noise for laser diodes with arbitrary amounts of optical feedback. IEEE Journal of Quantum Electronics 1998; 34(8): $1438-1446$. 
13. Ahmed M, Yamada Y. Field Fluctuations and Spectral Lineshape in Semiconductor Lasers Subjected to Optical Feedback. Journal of Applied Physics 2004; 95(12): 7573-7583.

14. Abdulrhmann S, Ahmed M, Yamada M. Analysis of dynamics and intensity noise of semiconductor lasers under strong optical feedback. Institute of Physics, Conference: Compound Semiconductors 2003; 147: 409-413.

15. Ahmed M, Yamada M. An infinite-order approach to gain calculation in injection semiconductor lasers. Journal of Applied Physics 1998; 84(6): 3004-3015.

16. Ahmed M, Yamada M, Saito M. Numerical modeling of intensity and phase noises in semiconductor lasers. IEEE Journal of Quantum Electronics 2001; 37(12): 16001610.

17. Ahmed M. Numerical approach to field fluctuations and spectral lineshape in InGaAsP laser diodes. International Journal of Numerical Modelling, Electronic Networks, Devices and Fields 2004; 17: 147-163.

18. Press WH, Teukolsky SA, Vetterling WT, Flannery BP. Numerical Recipes in Fortran: The Art of Scientific Computing. Cambridge University Press: New York, 1992.

19. Schunk N, Petermann K. Numerical analysis of the feedback regimes for a singlemode semiconductor laser with external feedback. IEEE Journal of Quantum Electronics 1988; 24(7): 1242-1247.

20. Mork J, Tromborg B, Mark J. Chaos in semiconductor laser with optical feedback: theory and experiment. IEEE Journal of Quantum Electronics 1992; 28(1): 93-108.

21. Kao YH, Wang NM, Chen HM. Mode description of routes to chaos in externalcavity coupled semiconductor lasers. IEEE Journal of Quantum Electronics 1994; 30(8): 1732-1739. 


\section{TABLE I}

TYPICAL VALUES OF THE PARAMETERS USED IN THE SIMULATIONS OF 980-nm InGaAs FIBER-GRATING LASERS

\begin{tabular}{ccc}
\hline Parameter & Value & Unit \\
\hline Slope of linear gain $a$ & $2.75 \times 10^{-12}$ & $\mathrm{~m}^{3} \mathrm{~s}^{-1}$ \\
Refractive index in fiber cavity $n_{e x}$ & 1.5 & -- \\
Length of fiber cavity $L_{e x}$ & varied & \\
Electron number at transparency $N_{g}$ & $1.89 \times 10^{8}$ & --- \\
Coefficient of nonlinear gain $B_{c}$ & $1.47 \times 10^{-6}$ & $\mathrm{~s}^{-1}$ \\
Electron life time $\tau_{s}$ & 2.79 & $\mathrm{~ns}$ \\
Linewidth enhancement factor $\alpha$ & 2 & -- \\
Refractive index of laser cavity $n_{D}$ & 3.5 & --- \\
Length of the active region $L_{\mathrm{D}}$ & 800 & $\mu \mathrm{m}$ \\
Volume of the active region $V$ & 400 & $\mu \mathrm{m}^{3}$ \\
Field confinement factor $\xi$ & 0.1 & --- \\
Reflectivity at the front facet $R_{f}$ & 0.02 & -- \\
Reflectivity at the back facet $R_{b}$ & 0.98 & -- \\
Threshold gain $G_{t h 0}$ of solitary laser & $2.11 \times 10^{-11}$ & $\mathrm{~s}^{-1}$ \\
Threshold current $I_{\text {th }}$ of solitary laser & 45 & $\mathrm{~mA}$ \\
\hline
\end{tabular}

Fig. 1. Schematic diagram of a FGSL. 
Fig. 2. Model of analysis of laser diode operating under OFB.

Fig. 3. Variations of the CW power $P_{G c}$ with (a) OFB strength $K_{e x}$ and (b) fiber cavity length $L_{\underline{F}}$, respectively, when $I=5 I_{t h \underline{0}}$. The periodic variation of $P_{G c} \underline{\text { with variation }}$

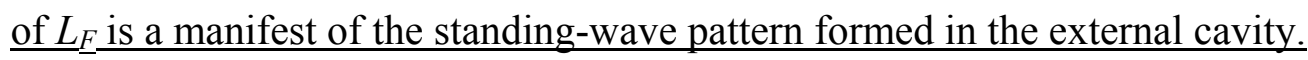

Fig. 4. Simulation results of short fiber cavity, $L_{F}=1 \mathrm{~cm}$ : (a)-(e) temporal trajectories of $S(t)$, and (f)-(j) RIN spectra when $K_{e x}=0.03(\mathrm{CW}), K_{e x}=0.07$ (pulsing), $K_{e x}=0.21$ (chaos), $K_{e x}=2.57$ (pulsing), $K_{e x}=3.0(\mathrm{CW})$, respectively.

Fig. 5. (a) Bifurcation diagram of $S(t)$, and (b) LF-RIN of a FGSL with short fiber cavity, $L_{F}=1 \mathrm{~cm}$. The operation is almost $\mathrm{CW}$ under weak and strong OFB having low RIN levels. When the laser operated in chaos, it exhibits most enhanced RIN. Under strong OFB, the laser happens to emit pulsation.

Fig. 6. Bifurcation diagrams of $S(t)$ under fiber cavities of length (a) $L_{F}=10 \mathrm{~cm}$, (b) $L_{F}=20 \mathrm{~cm}$, and (c) $L_{F}=80 \mathrm{~cm}$. The route-to-chaos becomes quasi-periodic for long fiber cavities. The dynamic instabilities promote with the increase of $L_{F}$.

Fig. 7. Simulation results of long fiber cavity, $L_{F}=20 \mathrm{~cm}$ : (a) and (c) temporal trajectories of $S(t)$, and (c) and (d) RIN spectra under strong OFB, $K_{e x}=3.1$ and 3.1, respectively. $K_{e x}$ corresponds to periodic pulsation with $f_{e x}$ and $K_{e x}$ corresponding to beating pulsation with $f_{e x}$ and $f_{r}$. 


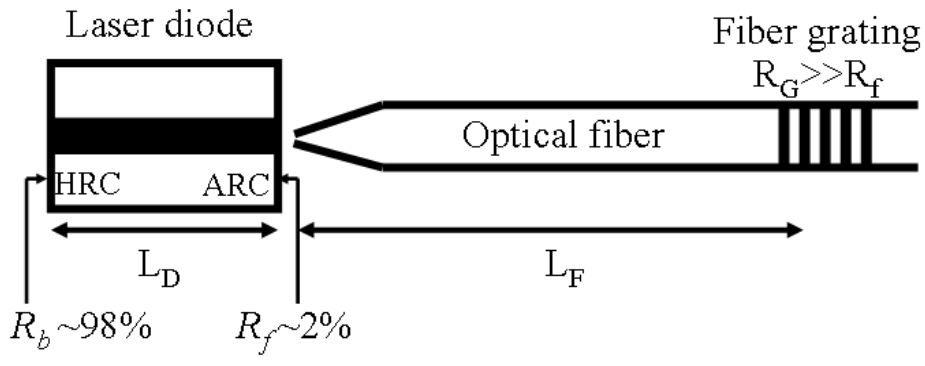

Fig. 1. 


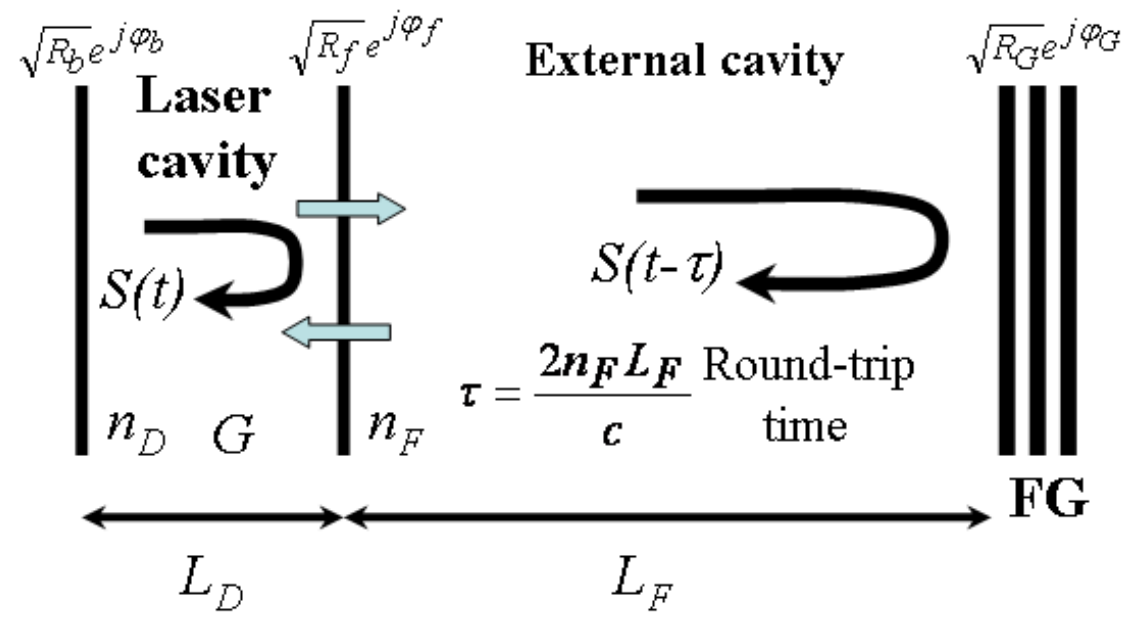

Fig. 2. 


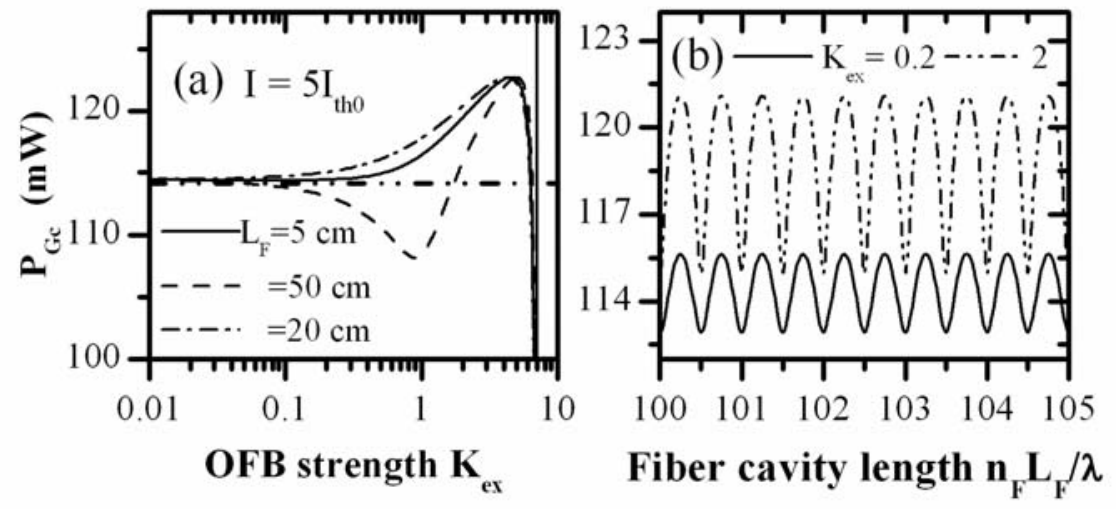

Fig. 3 


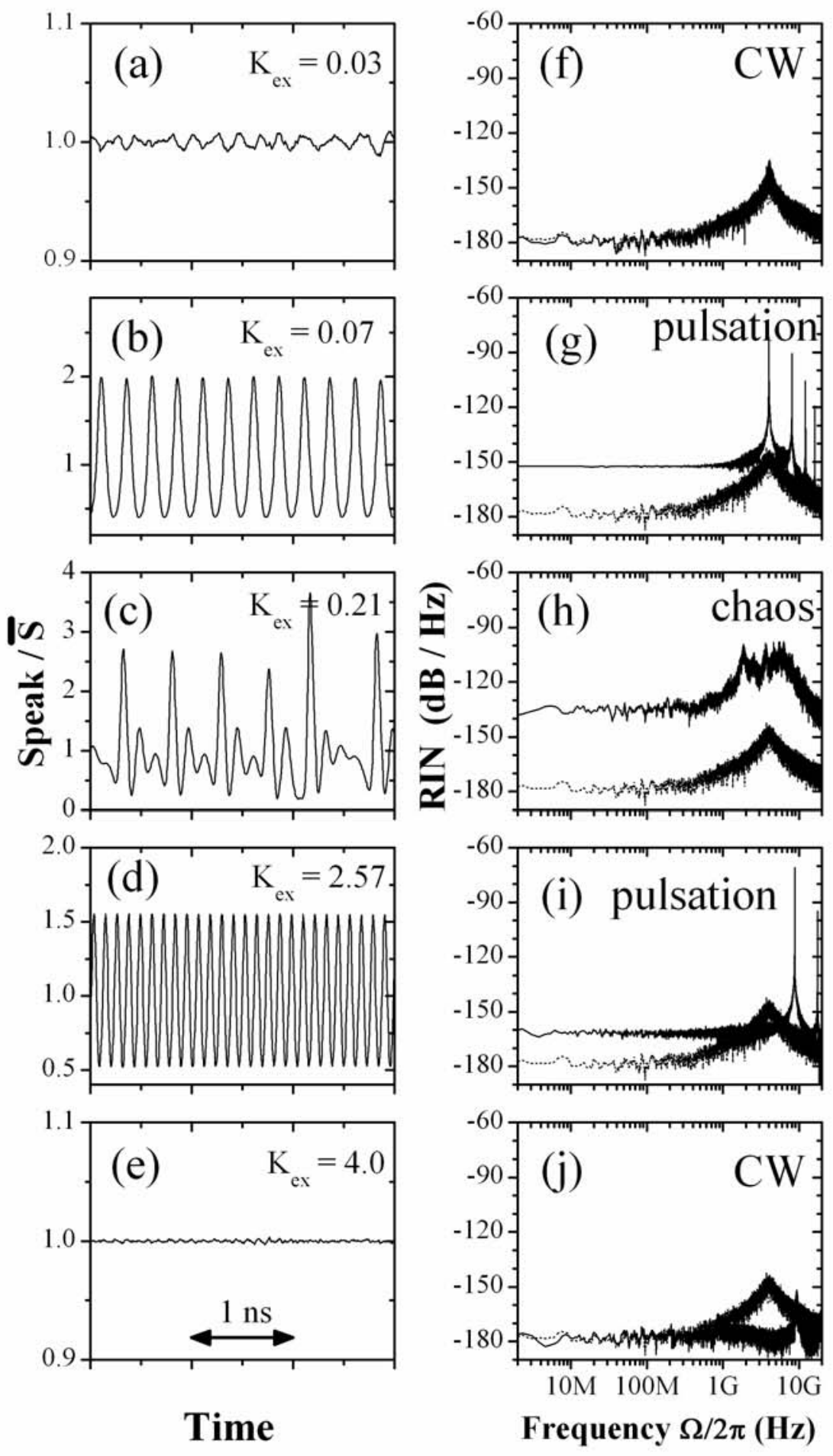

Fig. 4 


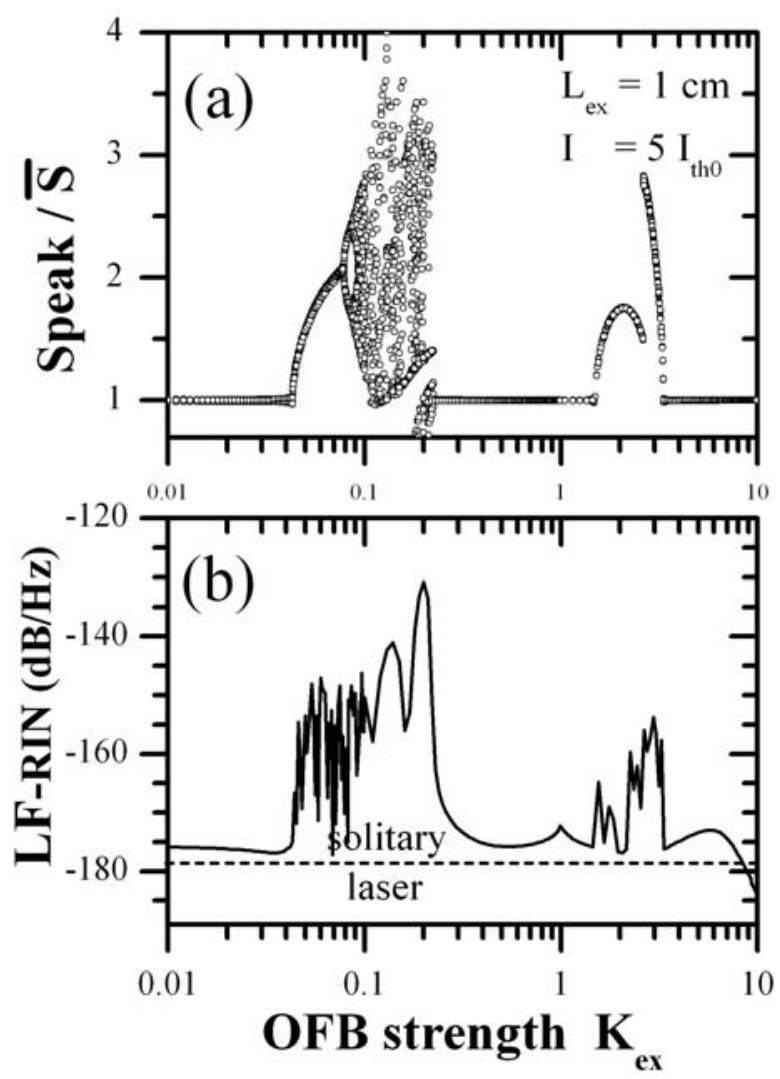

Fig. 5 


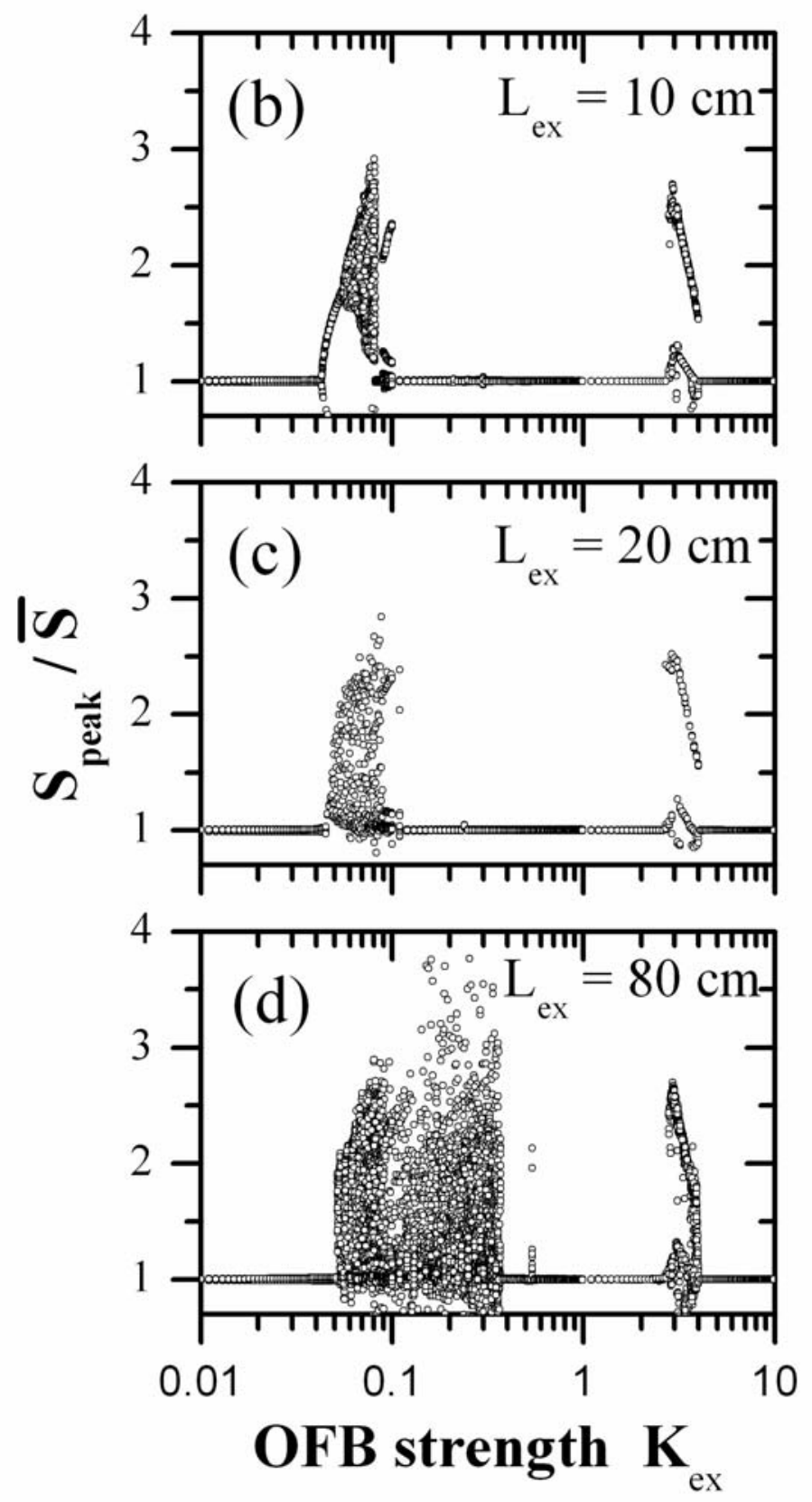

Fig. 6. 

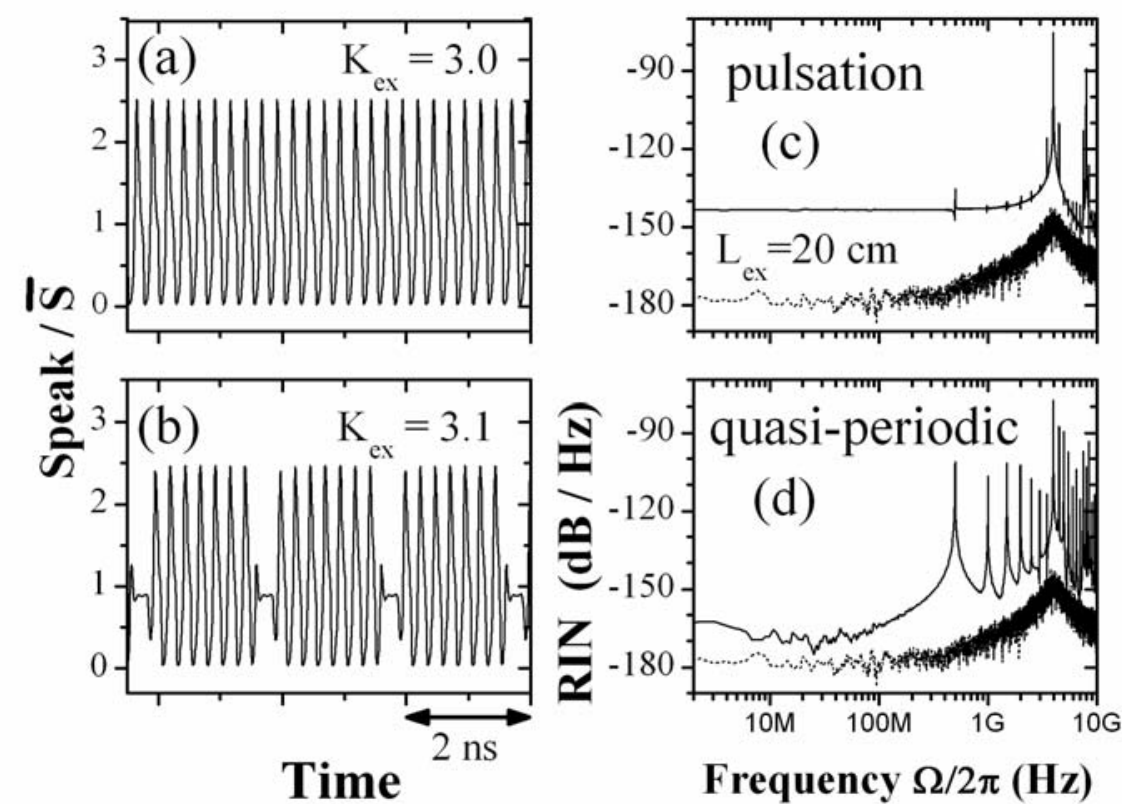

Fig. 7 